\title{
The mitochondrial A3243G mutation presenting as severe cardiomyopathy
}

\author{
Laura Vilarinho, Filippo M Santorelli, Maria J Rosas, Cláudia Tavares,
} Manuel Melo-Pires, Salvatore DiMauro

\begin{abstract}
A 6 year old Portuguese boy with dilated cardiomyopathy had abundant ragged red fibres in muscle (20\% of total) and severe lactic acidosis. Molecular genetic analysis showed the $A$ to $G$ transition in the mitochondrial transfer RNA ${ }^{\text {Leu(UUR) }}$ gene at nt 3243 ("MELAS mutation"), which accounted for $88 \%$ and $68 \%$ of the total mtDNA in his muscle and blood, respectively. Molecular studies in blood from 16 maternal relatives identified lower percentages of the mutation only in the oligosymptomatic mother and brother. This case reinforces the notion that cardiomyopathy. can be the presenting and predominant clinical expression of the A3243G mutation.

(F Med Genet 1997;34:607-609)
\end{abstract}

Keywords: mitochondrial DNA; A3243G; cardiomyopathy

Department of Clinica

Biology, Instituto de Genética Médica, $\mathbf{P r}$ Pedro Nunes 88, 4050

Porto, Portugal

L Vilarinho

Department of Neurology, College of Physicians and Surgeons, Columbia University, New York, NY, USA

L Vilarinho

F M Santorelli

S DiMauro

Department of Neurology, Hospital Senhora de Oliveira Guimarães, Portugal M J Rosas

Department of Paediatrics, Hospital Senhora de Oliveira, Guimarães, Portugal C Tavares

Division of Neuropathology, Hospital de S António, Porto, Portugal

M Melo-Pires

Correspondence to: Dr Vilarinho, Portugal.

Received 16 October 1996 Revised version accepted for publication 23 January 1997
Mitochondrial encephalomyopathy, lactic acidosis, and stroke-like episodes (MELAS) is a maternally inherited disorder first described by Shapira $e t a l^{1}$ in two sibs with normal development who presented between the ages of 3 and 4 years with growth retardation, hirsutism, and episodes of muscular weakness, anorexia, headache, and vomiting induced by exercise or intercurrent illnesses. MELAS was later recognised by Pavlakis $e t a l^{2}$ as an independent clinical syndrome. Clinical criteria for diagnosis have recently been revised by Hirano and Pavlakis $^{3}$ and include lactic acidosis, episodic vomiting, seizures, migraine-like headaches, short stature, and recurrent cerebral insults resembling strokes and causing hemiparesis, hemianopia, or cortical blindness. A point mutation in the $t R N A^{\text {Leu(UUR) }}$ gene of the human mitochondrial genome (A3243G) has been described $^{4}$ and has been found in the majority of patients with the clinical features of MELAS. However, both clinical and genetic heterogeneity are well documented.

We report the first Portuguese family harbouring the A3243G mutation, where the molecular defect was detected in a child with a severe dilated cardiomyopathy as the sole clinical manifestation. The present observation confirms the notion that mitochondrial DNA (mtDNA) mutations have variable clinical presentations and calls attention to the A3243G mutation as a cause of cardiomyopathy.
Case report

A 6 year old boy, born after an uneventful pregnancy to non-consanguineous parents, was admitted to hospital because of vomiting, general physical exhaustion, and unspecified muscle pain. He had developed normally until 3 years of age, when asthenia, anorexia, and poor physical growth were first noted. Episodes of postprandial vomiting without any other symptoms had occurred on several occasions and worsened over the next two years.

On admission, he was in physical distress but alert, and complained of generalised fatigue. Physical examination showed short stature; height was $110 \mathrm{~cm}$ and weight $20 \mathrm{~kg}$, which were both below the 5 th centile for age and sex. General examination showed a cardiac systolic bruit and echocardiography showed a dilated left ventricle. Long term electrocardiogram showed some episodes of sinus arrhythmia at night.

Neurological examination, brain CT scan, and electromyography were normal. In particular, there was no evidence of abnormal muscle tone, pyramidal tract signs, abnormal ocular muscle movements, or pigmentary retinopathy.

Metabolic investigation showed high levels of lactic acid $(6.2 \mathrm{mmol} / 1$ when fasted, $4.8 \mathrm{mmol} / 1$ when fed, normal $<2.5 \mathrm{mmol} / \mathrm{l}$ ) with an increased lactate/pyruvate ratio, low total carnitine levels $(27 \mu \mathrm{mol} / \mathrm{l}$, normal range: 35-70), and high creatine kinase (three times normal) and lactic dehydrogenase (five times normal).

Morphological studies of a skeletal muscle biopsy showed minimal atrophy and evidence of abnormal mitochondrial proliferation (ragged red fibres, RRF) with the modified Gomori trichrome stain. RRF accounted for more than $20 \%$ of the total fibres examined; most RRF showed strong oxidative activity with the histochemical stain for cytochrome-c oxidase (COX), but the same rare fibres were COX negative.

The family pedigree is shown in fig 1 . The family history was significant for the 36 year old mother, who suffered from an affective disorder and had attempted suicide in the past. The proband's 10 year old brother.was asymptomatic but mild hyperlactacidaemia was found in both the mother and brother $(2.8$ $\mathrm{mmol} / 1$ and $2.7 \mathrm{mmol} / 1$, respectively).

\section{Methods}

Spectrophotometric measurement of respiratory chain enzymes and citrate synthase were carried out in skeletal muscle homogenates as 


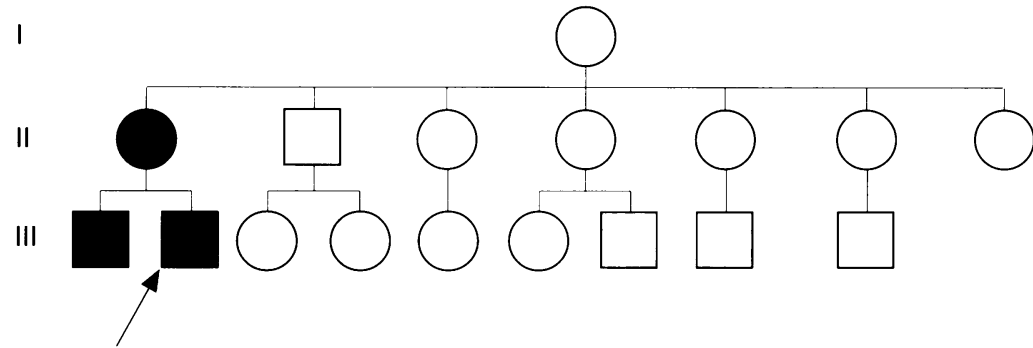

Figure 1 Family pedigree. Dark symbols show subjects who had detectable levels of the A3243G mutation. Arrow indicates the proband.

previously reported. ${ }^{5}$ High molecular weight DNA was extracted according to standard methods. Using oligonucleotide primers (5'-3') nt 3116-3134 and 3353-3333 (positions according to the published Cambridge sequence), we PCR amplified a 269 base pairs fragment encompassing the $16 \mathrm{~S}$ rRNA, tRNA ${ }^{\mathrm{Leu}(\mathrm{UUR})}$, and ND1 genes. PCR conditions were $94^{\circ} \mathrm{C}$ for one minute, $55^{\circ} \mathrm{C}$ for one minute, and $72^{\circ} \mathrm{C}$ for 45 seconds. A last "hot PCR cycle" was performed to avoid formation of heteroduplex molecules. ${ }^{6}$ Ten percent of PCR products were digested with the endonuclease HaeIII because the G at nt 3243 creates an additional site of cleavage in the mutated molecules. Fragments were separated through a $12 \%$ non-denaturing polyacrylamide gel and exposed to XAR-Kodak film at room temperature. To assess the abundance of the mutation, gels were scanned on a Phosphor-Imager (Biorad, Hercules, CA), and the results analysed by Image-Quant software (Molecular Dynamics, Sunnyvale, CA).

\section{Results and discussion}

Biochemical studies in our patient's muscle biopsy showed severe combined defects of the activities of complex I ( $20 \%$ normal), complex III $(22 \%)$, and complex IV (12\%), while citrate synthase was twice the value of normal controls. Southern blot analysis ruled out large scale $\mathrm{mtDNA}$ rearrangements.

MtDNA analysis showed the A3243G mutation (MELAS mutation) in $88 \%$ of the proband's total muscle mtDNA and in $68 \%$ of his blood mitochondrial genome. Lower percentages were detected in blood from the mother (43\%) and the older brother (49\%) while none of the other 14 maternal relatives tested positive in blood, the only tissue available for our investigation.

Although mitochondrial disorders associated with mtDNA defects are usually characterised by encephalomyopathy, these syndromes are multisystemic and virtually every organ system can be involved. ${ }^{7}$ Endocrinopathies such as diabetes mellitus, hypoparathyroidism, and growth hormone deficiencies are common. ${ }^{79}$ Renal tubular acidosis (de ToniFanconi Debré syndrome) is frequently associated with mitochondrial myopathies. Exocrine pancreatic dysfunction and sideroblastic anaemia are defining features of Pearson syndrome, which results from large scale mtDNA rearrangements. ${ }^{10}$ Mitochondrial patients have also presented with cardiomyopathy where cardiac dysfunction has three major manifesta- tions: cardiomyopathy (hypertrophic more often than dilated), cardiac conduction block, and pre-excitation syndrome. ${ }^{112}$ However, disorders dominated by heart disease are relatively rare and often present early in life as fatal infantile cardiomyopathies. ${ }^{1314}$

Our proband presented with a dilated cardiomyopathy as the predominant, if not the sole, clinical evidence of the A3243G mutation. The proportion of mutated genes was higher in muscle than in blood, it was less abundant in blood from the oligosymptomatic mother and brother, and it was not detected in blood of additional, healthy maternal relatives. Although the MELAS syndrome is usually characterised by CNS involvement and strokelike episodes before the age of 40 , there is considerable evidence of clinical heterogeneity, including familial progressive external ophthalmoparesis and maternally inherited diabetes mellitus and deafness. ${ }^{815}$ In this regard, our recent experience with more than 75 patients harbouring the A3243G mutation has shown that involvement of tissues other than the CNS is quite common (S Shanske, F M Santorelli, unpublished data). We also note that $20 \%$ of reported MELAS patients have had cardiomyopathy, which was hypertrophic in most cases, although cardiac symptoms were usually overshadowed by neurological manifestations. Our patient, therefore, illustrated yet another atypical presentation of the $\mathrm{A} 3243 \mathrm{G}$ mutation. It would not be surprising if additional phenotypes, including familial cardiomyopathies, were associated with this mutation. The affective disorder of the mother may also represent an atypical presentation of the A3243G mutation, as previously reported..$^{16-18}$

Although we did not detect significant levels of the A3243G mutation in blood from 14 additional maternal relatives, absence in blood does not preclude the possibility that postmitotic tissues, such as muscle and brain, may harbour detectable levels of mutated genes. However, these subjects' neurological and cardiological evaluations were normal. With the lack of efficient therapy, continuing monitoring of at risk subjects and genetic counselling are the only tools that we can now offer to this family.

We propose that mtDNA analysis should be included in the diagnostic approach to idiopathic dilated cardiomyopathies.

1 Shapira Y, Cederbaum SD, Cancilla PA, et al. Familial poliodystrophy, mitochondrial myopathy and lactic acidemia. Neurology 1975;25:614-21.

2 Pavlakis SG, Phillips PC, DiMauro S, et al. Mitochondrial myopathy, encephalopathy, lactic acidosis and strokelik myopathy, encephalopathy, lactic acidosis and strokelike 16:481-8.

3 Hirano M. Pavlakis S. Mitochondrial myopathy, encephalopathy, lactic acidosis, and strokelike episodes (MELAS) lopathy, lactic acidosis, and strokelike episodes

4 Goto Y-i, Nonaka I, Horai S. A mutation in the RNALeu(UUR) gene associated with the MELAS subgroup of mitochondrial encephalomyopathies. Nature 990;348:651-3.

5 DiMauro S, Servidei S, Zeviani M, et al. Cytochrome c ox dase in Leigh syndrome. Ann Neurol 1987;22:498-506.

6 Moraes CT, Ricci E, Bonilla E, DiMauro S, Schon EA. The mitochondrial tRNA ${ }^{\mathrm{Lu}(\mathrm{CLR})}$ mutation in MELAS: genetic, biochemical, and morphological correlations in skeletal muscle. Am f Hum Genet 1992;50:934-49.

7 DiMauro S, Hirano M, Bonilla E, DeVivo DC. The mitochondrial disorders. In: Berg O, ed. Principles of child neurology. New York: McGraw-Hill, 1996:1201-32. 
8 van den Ouweland JMW, Lemkes HP, Ruitenbeek W, et al. Mutation in mitochondrial tRNA $A^{\text {eu(UUR) }}$ gene in a large pedigree with maternally transmitted type II diabetes mellitus and deafness. Nat Genet 1992;1:368-71.

9 Hirano M, DiMauro S. Clinical features of mitochondrial myopathies and encephalomyopathies. In: Lane R, ed. Handbook of muscle disease. New York: Marcel Dekker, 1996:479-504.

10 McShane MA, Hammans SR, Sweeney M, et al. Pearson syndrome and mitochondrial encephalomyopathy in a patient with a deletion of mtDNA. Am $\mathcal{F}$ Hum Genet 1991; 48:39-42.

11 Anan $R$, Nakagawa $M$, Miyata $M$, et al. Cardiac involvement in mitochondrial diseases: a study on 17 patients with in mitochondrial diseases: a study on 17 patients with 91:955-61.

12 Williams R. Cardiac involvement in mitochondrial diseases and vice versa. Circulation 1995;91:1266-8.
13 Marin-Garcia J, Goldenthal MJ, Ananthakrishnan R, et al. Mitochondrial function in children with idiopathic dilated cardiomyopathy. F Inher Metab Dis 1996;19:309-12.

14 Tanaka M, Ino $\mathrm{H}$, Ohno $\mathrm{K}$, et al. Mitochondrial mutation in fatal infantile cardiomyopathy. Lancet 1990;ii: 1452.

15 Moraes CT, Ciacci F, Silvestri G, et al. Atypical clinical presentations associated with the MELAS mutation at position 3243 of human mitochondrial DNA. Neuromusc Disord 1993;3:43-50.

16 Damian MS, Seibel P, Reichmann H, et al. Clinical spectrum of the MELAS mutation in a large pedigree. Acta Nectrum of the MEand 1995;92:409-15.

17 Shanske AL, Shanske S, Silvestri G, et al. MELAS point mutation with unusual clinical presentation. Neuromusc Disord 1993;3:191-3.

18 Hammans SR, Sweeney MG, Hanna MG, et al. The tion. A clinical and genetic study. Brain 1995;118:721-34. 